\title{
Multiphoton excitation spectra in biological samples
}

\author{
Mary E. Dickinson \\ California Institute of Technology \\ Beckman Institute \\ Biological Imaging Center \\ Pasadena, California 91125 \\ E-mail: maryd@gg.caltech.edu

\section{Eva Simbuerger} \\ Bernhard Zimmermann \\ Carl Zeiss Jena $\mathrm{GmbH}$ \\ Advanced Imaging Microscopy Division \\ Jena, 07745 Germany

\section{Christopher W. Waters} \\ Scott E. Fraser \\ California Institute of Technology \\ Beckman Institute \\ Biological Imaging Center \\ Pasadena, California 91125
}

\begin{abstract}
Multiphoton microscopy is becoming a popular mode of live and fixed cell imaging. This mode of imaging offers several advantages due to the fact that fluorochrome excitation is a nonlinear event resulting in excitation only at the plane of focus. Multiphoton excitation is enhanced by the use of ultrafast lasers emitting in the near IR, offering better depth penetration coupled with efficient excitation. Because these lasers, such as titanium:sapphire lasers, offer tunable output it is possible to use them to collect multiphoton excitation spectra. We use the software-tunable Coherent Chameleon laser coupled to the Zeiss LSM 510 META NLO to acquire $x-y$ images of biological samples at multiple excitation wavelengths, creating excitation lambda stacks. The mean intensity of pixels within the image plotted versus excitation wavelength reveals the excitation spectra. Excitation lambda stacks can be separated into individual images corresponding to the signal from different dyes using linear unmixing algorithms in much the same way that emission fingerprinting can be used to generate crosstalk free channels from emission lambda stacks using the META detector. We show how this technique can be used to eliminate autofluorescence and to produce crosstalk-free images of dyes with very close overlap in their emission spectra that cannot be separated using emission fingerprinting. Moreover, excitation fingerprinting can be performed using nondescanned detectors (NDDs), offering more flexibility for eliminating autofluorescence or crosstalk between fluorochromes when imaging deep within the sample. Thus, excitation fingerprinting complements and extends the functions offered by the META detector and emission fingerprinting. We correct biases in the laser and microscope transmission to acquire realistic multiphoton excitation spectra for fluorochromes within cells using the microscope, which enables the optimization of the excitation wavelength for single and multilabel experiments and provides a means for studying the influence of the biological environment on nonlinear excitation. ๑ 2003 Society of Photo-Optical Instrumentation Engineers. [DOI: 10.1117/1.1583734]
\end{abstract}

Keywords: confocal microscopy; multiphoton microscopy; fluorescent proteins; fluorescence; multicolor imaging; excitation spectra.

Paper MM-13 received Mar. 5, 2003; revised manuscript received Mar. 25, 2003; accepted for publication Mar. 28, 2003.

\section{Introduction}

Multiphoton microscopy offers several advantages for imaging cells in living and fixed samples (see Refs. 1 and 2 for a review). The advantages of this technique result from the use of a near-IR laser to excite fluorochromes via a nonlinear process. Multiphoton excitation requires the quasisimultaneous absorption of multiple photons, usually two or three, to fulfill the energy requirement for dye excitation. To improve the efficiency of this process, ultrafast lasers emitting rapid pulse trains have been coupled to microscopes. Using this approach, very large peak intensities can be achieved in a repeated pulse train to sustain fluorophore excitation exclusively at the focal plane where the density of the photon flux is sufficiently high. While several laser sources have been utilized, titanium:sapphire lasers have become the most common light source for this application. Further developments in laser technology now include full automation and software control of titanium:sapphire lasers, resulting in turnkey laser sources for multiphoton microscopy.

In experiments where multiple dyes are used, spectral crosstalk can be a significant factor when using multiphoton excitation due to the relatively broad excitation spectra. Recently, we showed that linear unmixing is a powerful tool for eliminating spectral crosstalk between fluorochromes with overlapping emission spectra using laser scanning microscopy. ${ }^{3,4}$ Using the LSM 510 META, it is possible to produce crosstalk-free images of spectrally and spatially overlapping fluorochromes. ${ }^{4-7}$ This method, known as emission fingerprinting uses linear algebra to determine the relative contribution of individual overlapping spectral emission signals to the total signal in each pixel. The META detector is used to collect $x-y$ images at a series of emission wave-

1083-3668/2003/\$15.00 @ 2003 SPIE 
lengths. The known reference spectra for each of the multiple labels enables straightforward linear unmixing of the relative contributions using algorithms such as the singular value decomposition method. The characterization of the spectral components within a cell provides information about how emission signals can be collected most efficiently. In cases where considerable spectral overlap exists, this offers a means for crosstalk-free separation of fluorescent labels. In addition, using this approach it is possible to examine the effects of the environment on the dye being used and exploit these differences in experimental paradigms.

With this in hand, we turned our attention to excitation spectra. Excitation lambda stacks can be treated the same way as emission lambda stacks, enabling linear unmixing based on the variable excitation of fluorochromes at different wavelengths. Although multiphoton microscopy is now common in many laboratories, determining the peak excitation wavelength for individual experiments usually requires some guesswork. Two-photon cross-section data has been determined for some fluorochromes, ${ }^{8-12}$ but for other commonly used fluorochromes, trial and error has been the only way to determine the best excitation wavelength for peak excitation. The development of software-controlled, automated lasers makes it possible to employ strategies for rapidly determining the excitation spectra of a given dye. With these lasers coupled to the microscope it is then possible to determine the fluorescence produced at different excitation wavelengths within biological samples, offering another step toward understanding the relationship between dye structure, environment, and fluorescence.

In this paper, we show that we have developed techniques to acquire multiphoton excitation lambda stacks from biological samples on the microscope stage. Using a Coherent Chameleon laser controlled through the Zeiss LSM 510 META NLO software, we acquired lambda excitation stacks and we can unmix the relative components within these stacks using variations in the excitation spectra. Furthermore, we acquired excitation spectra in two ways. First, we acquired data where no effort was made to correct for the changes in power and pulsewidth at the sample as a result of tuning to different output wavelengths of the laser. These spectra show the optimum fluorescence generated at a particular excitation wavelength considering the gain curve of the laser and the transmission of the optics. Second, we corrected for uneven power at the sample with varying excitation wavelength to produce spectra that more faithfully reproduce data gleaned from true cross-section studies. Thus, the microscope user now has a way to optimize the excitation energy at the sample and can use the variations in excitation as a tool to eliminate crosstalk in samples with multiple labels.

\section{Materials and Methods}

\subsection{Cell Culture}

The tissue culture was HeLa cells (ATCC CL-2) and 3T3 cells (ATCC CCL-92) obtained from American Type Culture Collection (Manassas, Virginia). Cultures were maintained at $37^{\circ} \mathrm{C}$ in a water-jacketed incubator in $5 \% \mathrm{CO}_{2}$. Cells were grown in Dulbeccos Modified Eagles Media High Glucose (Irvine Scientific \#9031) supplemented with $1 \times$ penicillin/ streptomycin, $1 \times 1=$ Glutamine, and $10 \%$ heat-inactivated fetal bovine serum. Cells were cultured on circular cover slip glass (\#1) to 70 to $80 \%$ confluency.

\subsection{Cell Transfection}

Fluorescent proteins were introduced into cells by transfection or viral infection. Transfection of GFP Expressing Plasmid (gWIZ, GTS, San Diego, California) using Lipomer transfection reagent was performed essentially as previously described. ${ }^{13}$ The incubation media was replaced with media containing $2 \mu \mathrm{l} / 1 \mathrm{ml}$ of transfection reagent and cells were incubated for 12 to $14 \mathrm{~h}$. H2B-CFP, H2B-GFP, and H2B-YFP fusion proteins were introduced into $3 \mathrm{~T} 3$ cells via viral infection as previously described in Ref. 1 .

\subsection{Cell and Tissue Staining}

Drosophila eyes were prepared as whole mount samples and fixed in $3 \%$ paraformaldehyde in phosphate buffer $(0.1 \mathrm{M}, \mathrm{pH}$ 7.4) for $1 \mathrm{~h}$ at room temperature. Following three washes in PBS, samples were incubated with Alexa Fluor 568Phalloidin (A-12380 Molecular Probes) in PBS, 0.1\% Triton $\mathrm{X} 100$ for $16 \mathrm{~h}$ at room temperature. The samples were washed three times in PBS and mounted using Mowiol.

Unstained or GFP transfected cells grown on cover slips were fixed in $4 \%$ paraformaldehyde for $1 \mathrm{~h}$ at room temperature prior to staining.

Hoechst 33342 (H-3570 Molecular Probes, Eugene, Oregon) was used to stain cells at a concentration of $1 \mu \mathrm{g} / \mathrm{ml}$ for $5 \mathrm{~min}$, followed by three $10-\mathrm{min}$ washes in PBS solution. Cells were postfixed for $1 \mathrm{~h}$ with $4 \%$ paraformaldehyde at room temperature followed by three washes with PBS.

Sytox Green was used to stain nuclei (S-7020 Molecular Probes) at a concentration of $5 \mathrm{mM}$ (a 1:5000 dilution from stock) for 10 to $30 \mathrm{~min}$, followed by three 5-min washes in PBS solution. Cells were postfixed for $1 \mathrm{~h}$ with $4 \%$ paraformaldehyde at room temperature followed by three washes with PBS.

Fluorescent phalloidins conjugated to either Fluorescein (F-432 Molecular Probes), Rhodamine (R-415, Molecular Probes), Alexa Fluor 488 (A-12379 Molecular Probes), Alexa Fluor 546 (A-22283 Molecular Probes), Alexa Fluor 568 (A12380 Molcular Probes), Alexa Fluor 594 (A-12381 Molecular Probes), or Alexa Fluor 633 (A-22284 Molecular Probes) were added to fixed cells at a concentration of $0.67 \mu \mathrm{M}$ (a 1:10 dilution of the stock solution in PBS) for $30 \mathrm{~min}$. Samples were then rinsed in PBS and postfixed for $1 \mathrm{~h}$ at room temperature before mounting.

All samples were mounted using ProLong mounting media (P-7481 Molecular Probes, Eugene, Oregon) or a Mowiol/ glycerol mixture.

\subsection{Image Collection and Analysis}

All images and image stacks were collected using a Zeiss LSM 510 META NLO mounted on an Axiovert 200M inverted microscope (Carl Zeiss Microimaging, Inc., Thornwood, New York) and coupled to a Coherent Chameleon tunable titanium:sapphire laser (Coherent Laser Group, Santa Clara, California). Images were collected using a Zeiss PlanNeofluar $40 \times / 1.3$ numerical aperture (NA) objective lens except for the comparison data shown in Fig. 5(a) in Sec. 3.2, 
where a Zeiss IR-Achroplan $63 \times / 0.95$ NA objective was used. All images are $512 \times 512$ pixels in size and have a 12 -bit pixel depth. For image acquisition, linear unmixing was performed using the Zeiss LSM 5 version 3.2 software. Excitation lambda stacks were acquired using a macro to synchronize the task of tuning the laser and acquiring the image.

\subsection{Measurements for Bias Correction}

Several measurements were made to determine the origin and extent of the bias effecting dye excitation. First, we examined the reproducibility of the power of the laser by tuning to the same wavelength series, 720 to $940 \mathrm{~nm}$ in 20-nm increments. Eleven trips were made through this tuning range on six different days over a month-long period. Power was measured using a Coherent Fieldmaster GS with an LM-10detector head (Coherent Inc., Santa Clara, California). The mean and standard deviations from these measurements are shown in Fig. 4(a). Power measurements taken in a similar way were recorded at the objective. For these measurements, a semiconductor power meter was used to accurately measure low power levels [Field master GS with LM-2 VIS (400 to 1064 nm) detector head; Coherent Inc., Santa Clara, California]. Six trips through the tuning range were made and the mean and standard deviations from these measurements are shown in Fig. 4(b). For these measurements, single-direction scanning (the beam is blanking on the flyback) was used at a scanner zoom of 40 using $10 \%$ transmission through the acousto-optic modulator (AOM). The pulsewidth at the sample was characterized using an autocorrelator designed to measure pulsewidth through the objective lens (Carpe, APE, Berlin, New Jersey, and INRAD, Northvale, New Jersey). The laser was tuned through the 720 to $940-\mathrm{nm}$ range in $20-\mathrm{nm}$ increments five times. Measurements through the objective were fitted to a $\operatorname{sech}^{2}$ distribution to determine the actual pulsewidth. Figure 4(c) shows the mean pulsewidth measurement related to wavelength including the standard deviation.

\subsection{Correction for Power at the Sample}

To determine the attenuation settings required to produce the same power at the sample for each wavelength, power measurements were taken at the sample from 0 to $10 \%$ attenuation in $1 \%$ increments. The power measurements were plotted with respect to percentage of attenuation and fitted to a polynomial regression, which provided the best fit for these values. For each fluorochrome, an excitation lambda stack was acquired with no bias correction to get a general idea where the peak would be. This peak wavelength served as a starting point for determining the best power level and detector gain setting for efficient detection of fluorescence. For each of the other 11 wavelength points, the percentage of attenuation required for equivalent power was calculated using the curves generated from the power measurements taken at each wavelength. Once the power measurements are established, a look-up table can be used to adjust laser attenuation to acquire corrected excitation stacks for any dye. To determine the efficacy of this approach the power was directly measured at the calculated percentages of attenuation derived for each wavelength. We found that the power measured at these settings was within $4 \%$ of the target power value (data not shown). Using the attenuation values established for each wavelength, $x-y$ im- ages were acquired maintaining the same detector gain setting. Shifts in the focal plane due to spherical aberrations were corrected either by using an autofocus macro or by adjusting the collimator lens to predefined settings for each wavelength. For each dye, a minimum of three fields of view was imaged for each wavelength series. The average intensities from 10 regions of interest (ROIs) were determined to produce the curves shown in Fig. 5 in Sec. 3.2. To prevent bleaching, we minimized the amount of power required to collect each series and then collected data in both the 720- to 940-nm direction and then from 940 to $720 \mathrm{~nm}$. For all the data that were acquired, bleaching was limited to less than $5 \%$ of the average intensity (data not shown).

\section{Results and Discussion}

\subsection{Eliminating Spectral Crosstalk Using Excitation Eingerprinting}

Previously, we and others have shown that the LSM 510 META can be used to resolve overlapping emission spectra in laser scanned images using linear unmixing, a process known as emission fingerprinting. ${ }^{4-7}$ In fact, using the LSM 510 META, dyes with as little as a few nanometers of separation in emission profiles can be unmixed. ${ }^{4,5}$ Given the success of this approach, we were eager to establish a means to use excitation spectra in a similar way. To accomplish this goal, we utilized the Coherent Chameleon laser as a tunable excitation source. This laser can be controlled via software commands conveyed through an RS232 connection. This enabled us to synchronize the computer-controlled LSM 510 META scan head with the laser via a simple macro. The wavelength range and interval are specified in the macro control window, and as the laser tunes to each wavelength an image is captured producing an excitation lambda stack. Figure 1 (see Color Plate 1) shows a comparison between excitation and emission fingerprinting for two commonly used green dyes, eGFP and Alexa Fluor 488. In this example, eGFP is localized to the nucleus using a construct expressing a histone 2B-eGFP fusion. Cells are then fixed and counterstained with Alexa Fluor 488 Phalloidin, which binds actin. Figure 1(a) shows the excitation lambda stack where images were collected in 20-nm intervals from 720 to $940 \mathrm{~nm}$ of excitation. Figure 1(b) shows the emission lambda stack for images collected in 10-nm intervals from 500 to $580 \mathrm{~nm}$ of emission using 780-nm excitation. Figure 1(c) shows the reference spectra defined for eGFP and Alexa Fluor 488 for both excitation (solid line) and emission (dashed line) fingerprinting. As expected, the multiphoton excitation curves are broad and the peaks do not correspond to twice the single-photon excitation peaks. Similar results were obtained using both excitation [Fig. 1(d)] and emission [Fig. 1(e)] fingerprinting. Thus, the same linear unmixing algorithms applied to emission fingerprinting using META detection can be used for crosstalk elimination using excitation fingerprinting.

Since multiphoton excitation results in broad curves with unpredictable peaks, it is possible that dyes with very similar and overlapping emission curves will have distinct multiphoton excitation curves with less overlap than in the emission curves. Therefore, it may be possible to use excitation fingerprinting to separate dyes that have too much spectral overlap for reliable unmixing using emission fingerprinting. We found 
A.

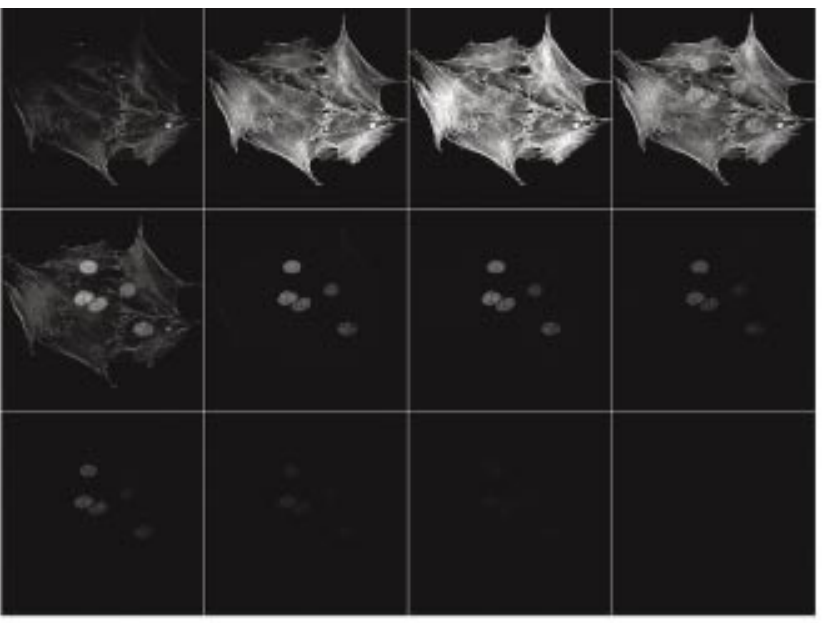

B.

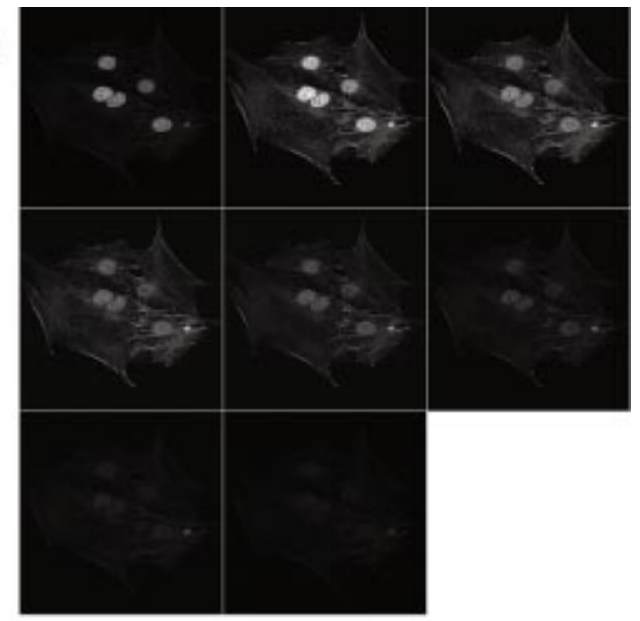

C.

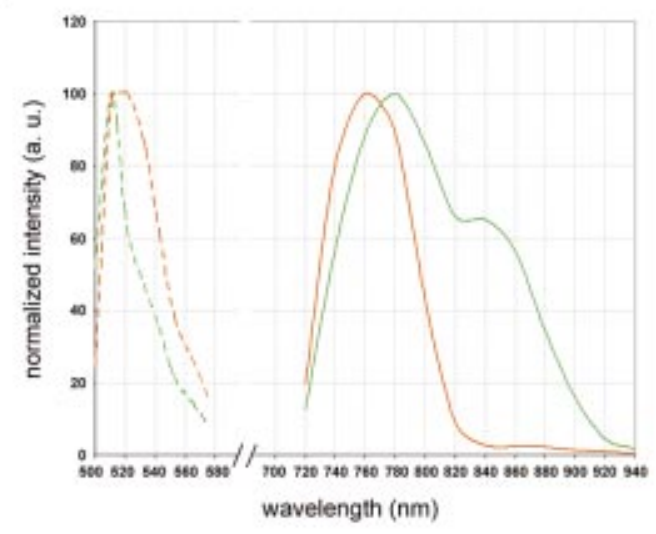

D.

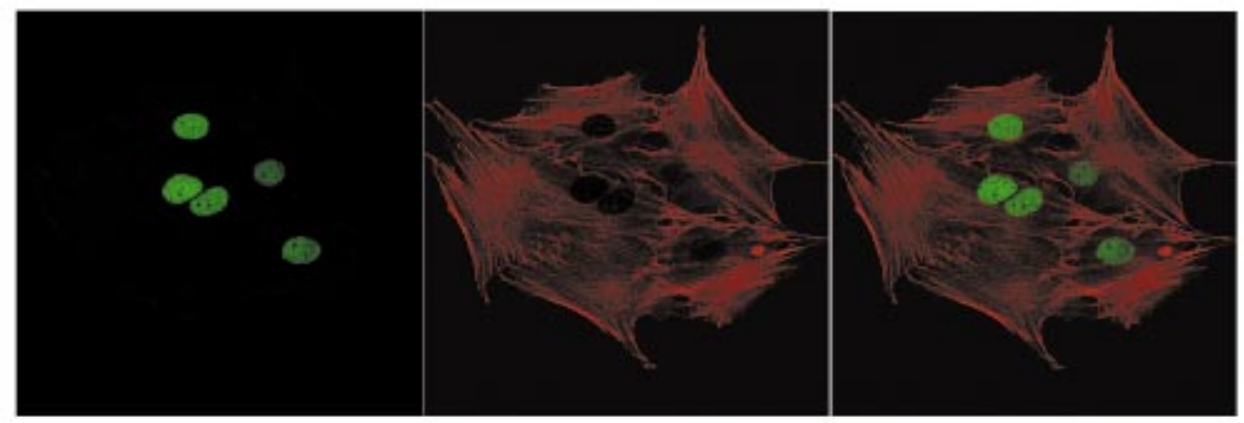

E.

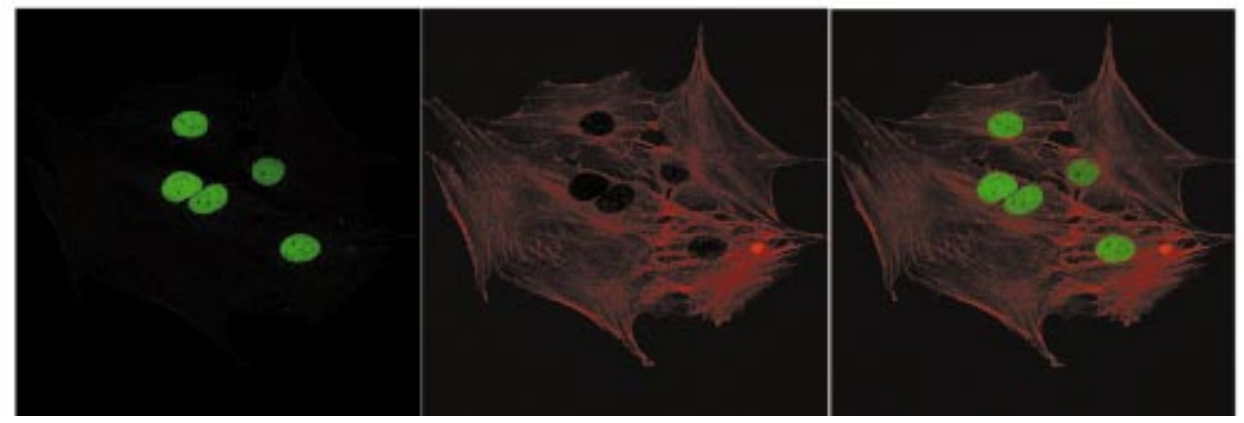

Fig. 1 Comparison of excitation and emission fingerprinting of 3T3 cells labeled with H2B-eGFP and Fluorescein Phalloidin: (a) the excitation lambda stack from 720 to $940 \mathrm{~nm}$ taken at 20-nm intervals using channel 2 and a BP500-550IR filter, (b) the emission lambda stack acquired with the META detector from 495 to $580 \mathrm{~nm}$ at 10.7-nm intervals, (c) the emission reference spectra for eGFP (dashed green line) and Alexa Fluor 488 (dashed red line) and the excitation reference spectra for eGFP (solid green line) and Alexa Fluor 488 (solid red line), and results of (d) excitation fingerprinting and (e) emission fingerprinting. In each case the first panel (green) is the H2B-GFP signal, the second panel (red) is Alexa Fluor 488 Phalloidin, and the third panel (red and green) is the overlay image.

332 Journal of Biomedical Optics • July 2003 • Vol. 8 No. 3 


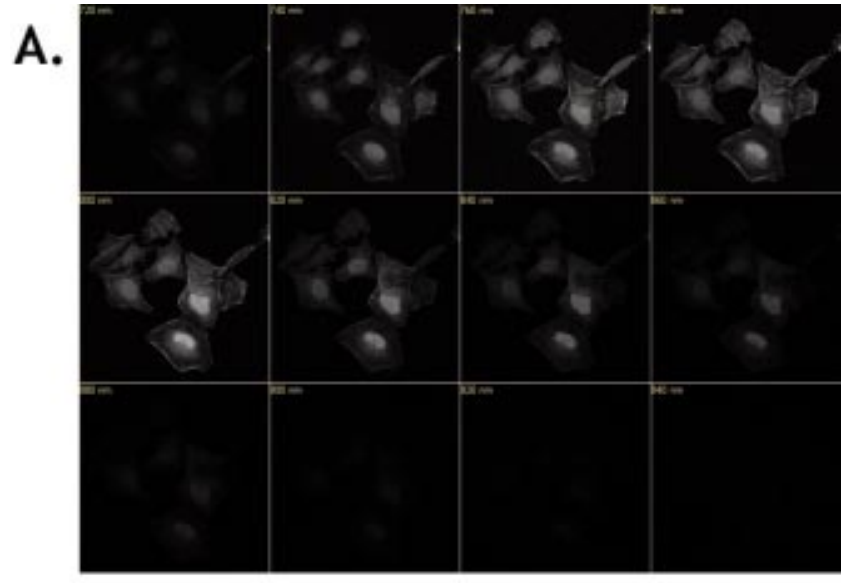

B.
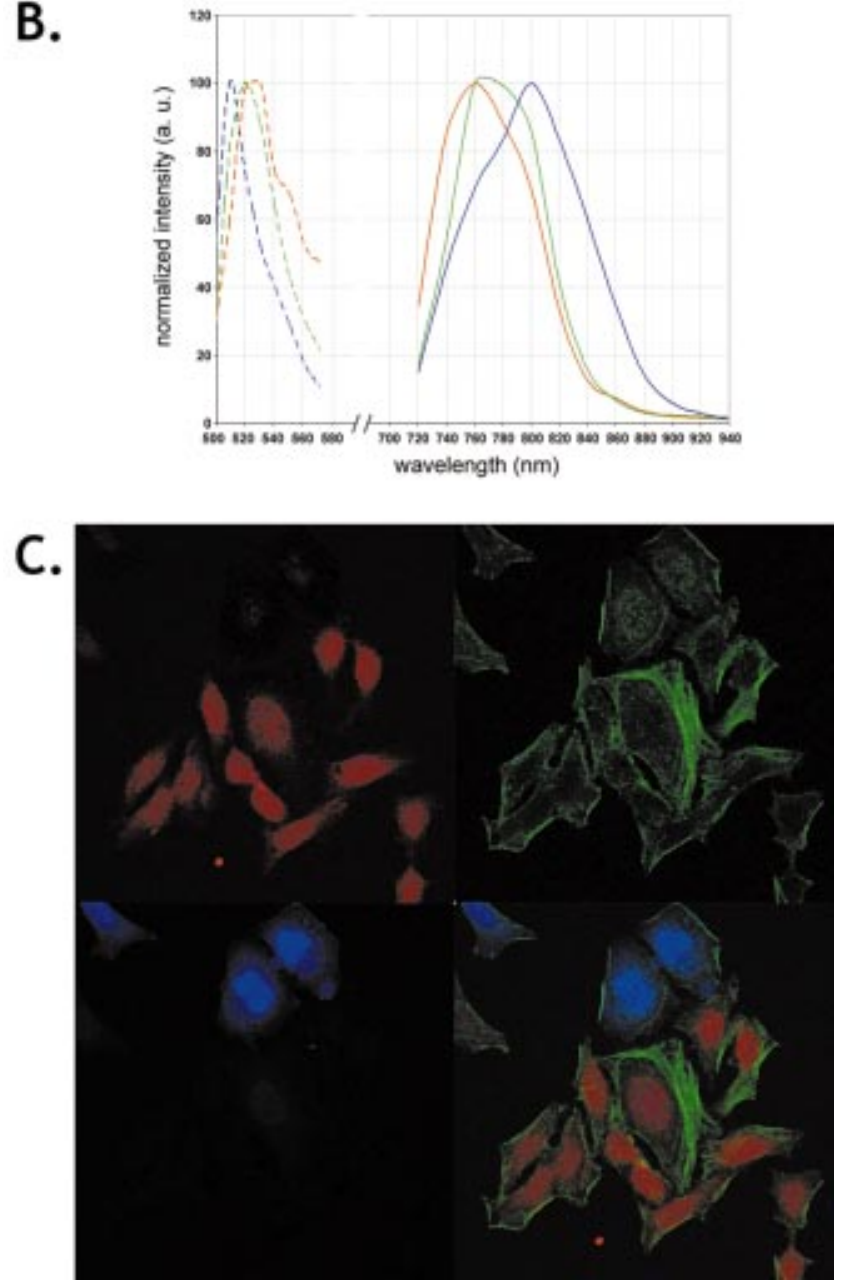

Fig. 2 Excitation fingerprinting of 3T3 cells labeled with Sytox Green, Fluorescein Phalloidin and eGFP: (a) the excitation lambda stack containing images taken from 720 to $940 \mathrm{~nm}$ in 20-nm increments using channel 2 and a BP500550 IR filter; (b) the emission spectra for Sytox Green (red dashed line), Fluorescein Phalloidin (green dashed line), and eGFP (blue dashed line) and the excitation spectra for Sytox Green (red solid line), Fluorescein Phalloidin (green solid line) and eGFP (blue solid line) used for excitation fingerprinting; and (c) the result of the linear unmixing algorithm using excitation fingerprinting, crosstalk-free separation of the three fluorochromes, Sytox Green (red), Fluorescein Phalloidin (green), and eGFP (blue). Emission fingerprinting with these three dyes leads to unreliable results (data not shown).
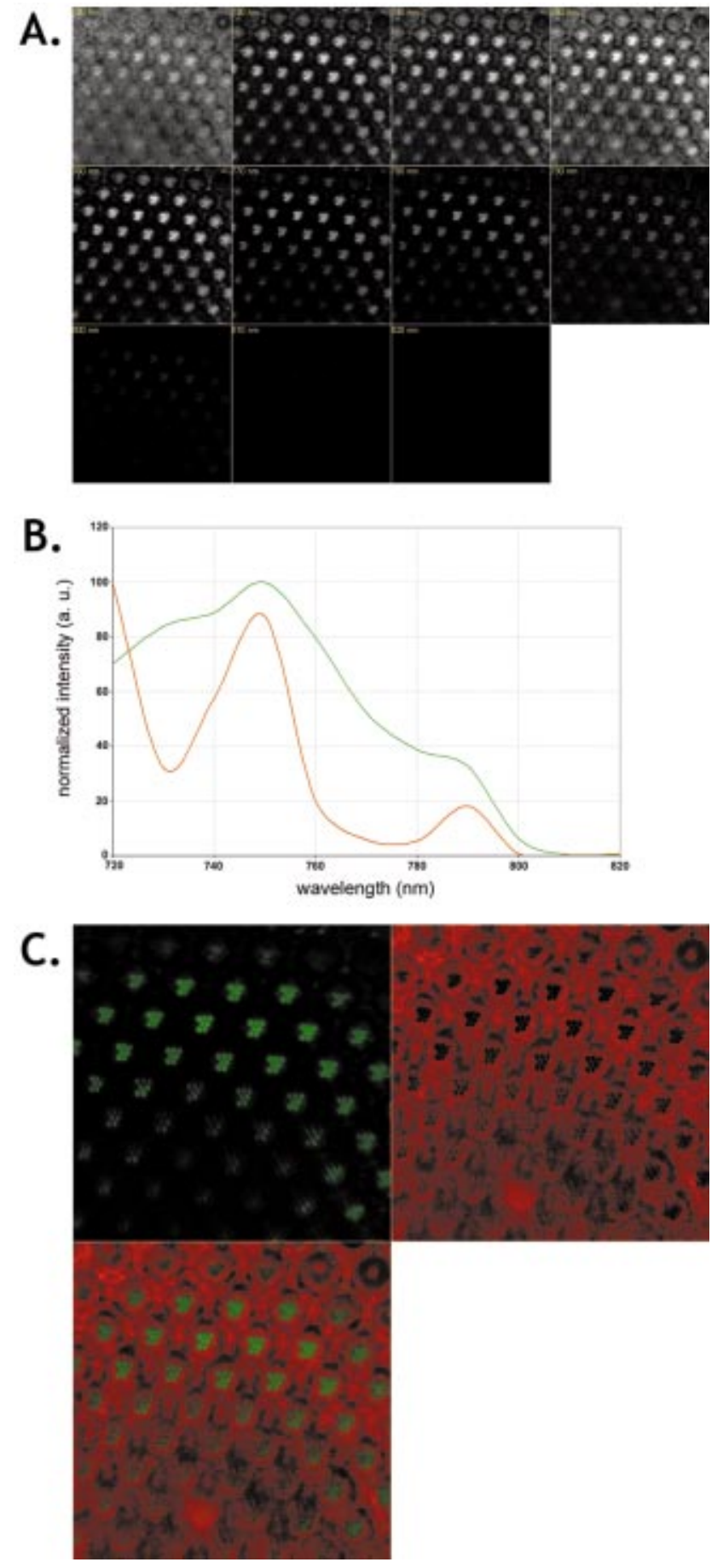

Fig. 3 Excitation fingerprinting of a highly autofluorescent sample, the Drosophila retina, which is also labeled with Alexa Fluor 568 Phalloidin: (a) the excitation lambda stack containing images taken from 720 to $820 \mathrm{~nm}$ in 10-nm increments; (b) the reference spectra for Alexa Fluor 568 Phalloidin (green) and the autofluorescence (green) used for linear unmixing; and (c) the result of the linear unmixing algorithm, crosstalk-free separation of the specific label Alexa Fluor 568 Phalloidin (green) and the autofluorescence signal (red). 

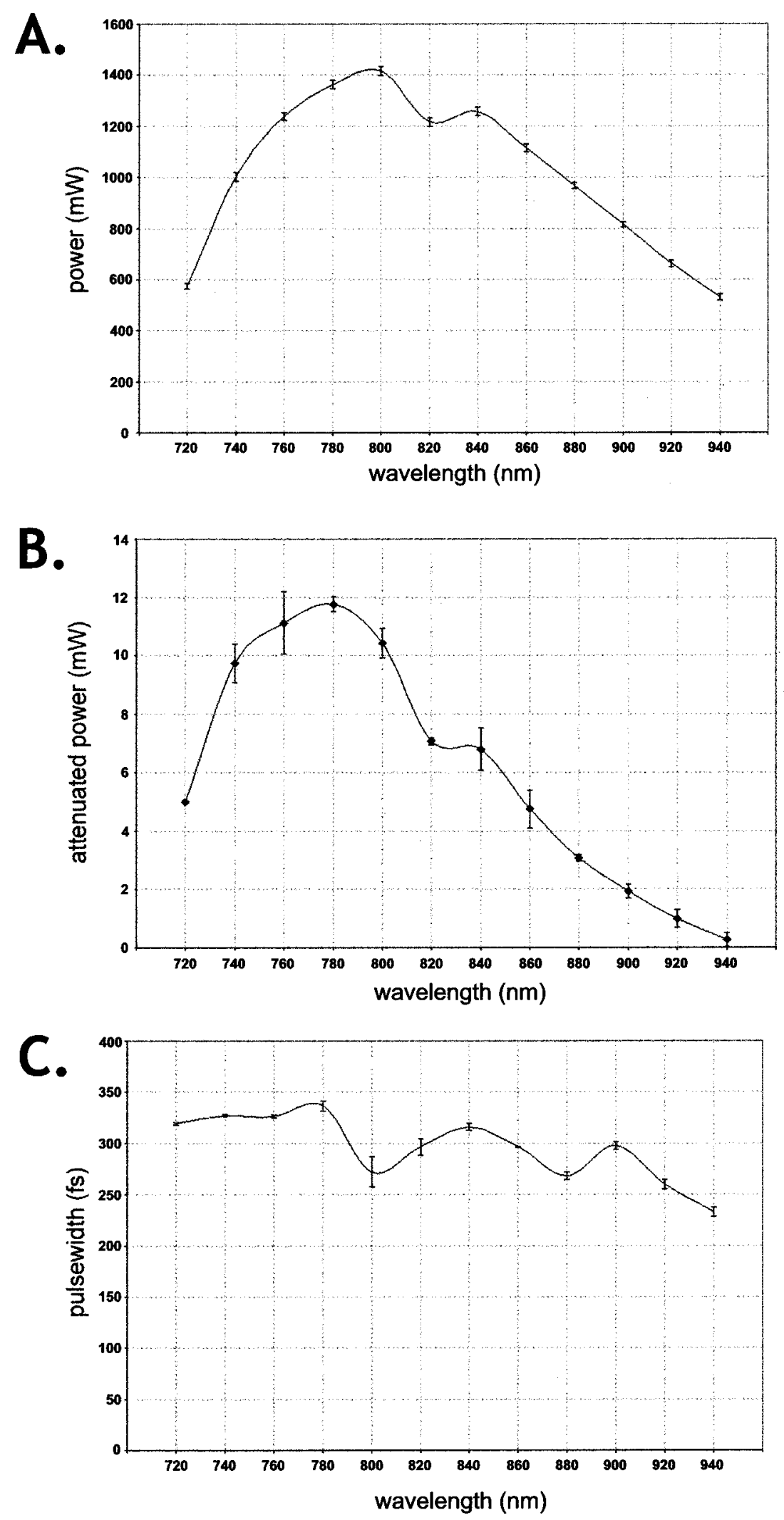

Fig. 4 Characterization of the power and pulsewidth of the excitation source: (a) the mean power at the laser head from 720 to $940 \mathrm{~nm}$ taken in 20-nm increments, where measurements were made repeated over several days to establish the reproducibility of laser power (see Sec. 2); (b) the mean power at the sample through the Plan-NeoFluar $40 \times 1.3-N A$ objective lens measured as described in Sec. 2; and (c) the mean pulsewidth measured at the sample with the Carpe autocorrelator using the Plan-NeoFluar 40×1.3-NA objective lens. Less than 100 fs difference in the average pulsewidth is seen at the sample. 
this to be true when using Sytox Green, Fluorescein, and eGFP. Figure 2(a) (see Color Plate 2) shows an excitation lambda stack where images were collected in 20-nm intervals from 720 to $940 \mathrm{~nm}$ of excitation. These three dyes, Sytox Green, GFP and FITC, have very closely overlapping emission spectra ${ }^{5}$ [Fig. 1(b) dashed lines], but the two-photon excitation spectra show distinct curves [Fig. 2(b), solid lines]. Although it was not possible to use linear unmixing to separate these three dyes reliably using emission fingerprinting ${ }^{5}$ (data not shown), crosstalk-free images of dye localization could be easily produced using excitation fingerprinting [Fig. 2(c)]. Sytox Green is shown in the red channel, FITC in the green channel, and GFP in the blue channel. Thus, we show that it is possible to extend the capabilities offered by emission fingerprinting when excitation fingerprinting is used.

Excitation spectra are also useful in separating a fluorescent label from autofluorescence in a sample. Autofluorescence is often strongly excited using multiphoton microscopy. We used wildtype Drosophila, which show a strong autofluorescent signal when excited with a near-IR (NIR) source, to demonstrate the feasibility of excitation fingerprinting for this application. Retinae stained for actin using Alexa Fluor 568 Phalloidin showed both strong autofluorescent and fluorescent signals. An excitation lambda stack was acquired tuning the laser from 720 to $820 \mathrm{~nm}$ in 10-nm steps. Figure 3(a) (see Color Plate 2) is the excitation lambda stack, indicating different intensity values for the autofluorescence and the fluorescent label. Excitation spectra were defined manually by choosing the appropriate points in the image stack [Fig. 3(b)]. These spectra were then used to perform linear unmixing. The result is shown in Fig. 3(c). There is a clear separation of autofluorescence (shown in red) and the stained actin filaments in the rhabdomeres of the photoreceptor cells (green).

Similar to the results obtained using emission fingerprinting, we were able to use excitation fingerprinting to selectively separate the fluorescent signal from overlapping fluorochromes or autofluorescent signals. For emission fingerprinting, we have utilized the LSM 510 META, which enables the simultaneous acquisition of $X-Y$ images at multiple wavelength intervals. For excitation fingerprinting, we have taken advantage of the software-controlled tuning of the Chameleon titanium:sapphire laser. For this approach, our acquisition is sequential and, therefore, slower than lambda stack acquisition for emission signals using the META detector. An excitation lambda stack consisting of data from 12 different wavelengths across the tunable spectrum of the laser was acquired in less than $2 \mathrm{~min}$. This makes it a reasonable approach for crosstalk separation in many samples. In addition, excitation lambda stacks can be acquired using any detector on the system, including the descanned detectors, nondescanned detectors, or even the META detector. Thus, this represents an approach that can be used for efficient crosstalk separation in deep scattering tissue, in conjunction with emission bandpass filters, or over a specified range of the META detector.

\subsection{Acquiring Unbiased Multiphoton Excitation Spectra from Biological Samples Using the Laser Scanning Microscope}

We already showed that a tunable titanium:sapphire laser can be used to acquire excitation lambda stacks that can be used for excitation fingerprinting to eliminate spectral crosstalk. However, spectral information acquired in this way is subject to considerable bias. This results from three factors. First, titanium:sapphire lasers have variable power output across the tuning spectrum. Second, the pulsewidth across this tuning range varies with respect to wavelength. Last, the transmission of many microscope optics are not uniform over this range of the laser. Most objectives decrease in transmission with increasing wavelength, further influencing the bias in excitation energy at the sample with respect to wavelength. Multiphoton excitation is a nonlinear process. The relationship between power, pulsewidth, and excitation probability can be stated as

$$
n_{a} \alpha \delta\left[\left(P_{\mathrm{av}}^{2} / \tau\right) f^{2}\right]\left[\pi\left(\mathrm{NA}^{2} / h c \lambda\right)\right]^{2},
$$

where the $n_{a}$ is the probability of excitation, $\delta$ is the excitation crosssection of a dye, $P_{\text {av }}$ is the average power, $\tau$ is the pulsewidth, $f$ is the repetition frequency of the pulse train from the laser, NA is the NA of the lens, $h$ is Planck's constant, $c$ is the speed of light, and $\lambda$ is the wavelength (see also Ref. 14). Although spectral information biased by these factors is useful, proper experimental optimization requires being able to resolve nonbiased spectra. Moreover, the drop-off in power as the laser wavelength increases, limits the ability to resolve peaks at higher wavelengths.

To acquire nonbiased excitation spectra, we reasoned that if the laser power and pulsewidth values were reproducible, we could vary the power at the sample with respect to wavelength to normalize the bias imposed by the source and the optics. Thus, we measured power and pulsewidth through repeated tunings across the extent of the wavelength range of the Chameleon to determine reliability and to gather measurements for corrections (Fig. 4). These data show that power levels with respect to wavelength are very reproducible, both at the laser head [Fig. 4(a)] and at the objective [Fig. 4(b)]. Clearly, there is a distinct bias with maximum power at the sample around $770 \mathrm{~nm}$ and decreasing over $70 \%$ between 780 and $940 \mathrm{~nm}$. Pulsewidth measurements made at the sample were also highly reproducible with repeated tuning [Fig. 4(c)]. Interestingly, the average variation in pulsewidth across the tuning range at the sample is less than $100 \mathrm{fs}$. Given the relationship between pulswidth and power, and the small variation in pulsewidth, the changes in power at the sample that would be required to compensate for the variation in pulsewidth are very small. Therefore, we neglected this correction in our calculations at present. Clearly the greatest influence on dye excitation is the variation in power with respect to wavelength.

To compensate for power at the sample we measured power at each wavelength with different levels of laser attenuation. The LSM 510 META NLO utilizes an AOM to attenuate laser power. The transmission through the AOM (percentage of diffraction into the first order) is linear over the entire range of attenuation values. Since we are using only a small fraction of the laser power for excitation, we measured the power at the sample between 0 and $10 \%$ attenuation. The power versus attenuation values for this range best fit a polynomial regression (data not shown). Using these data we can easily calculate the percentage of transmission needed at each wavelength to normalize power at the sample (see Sec. 2). 

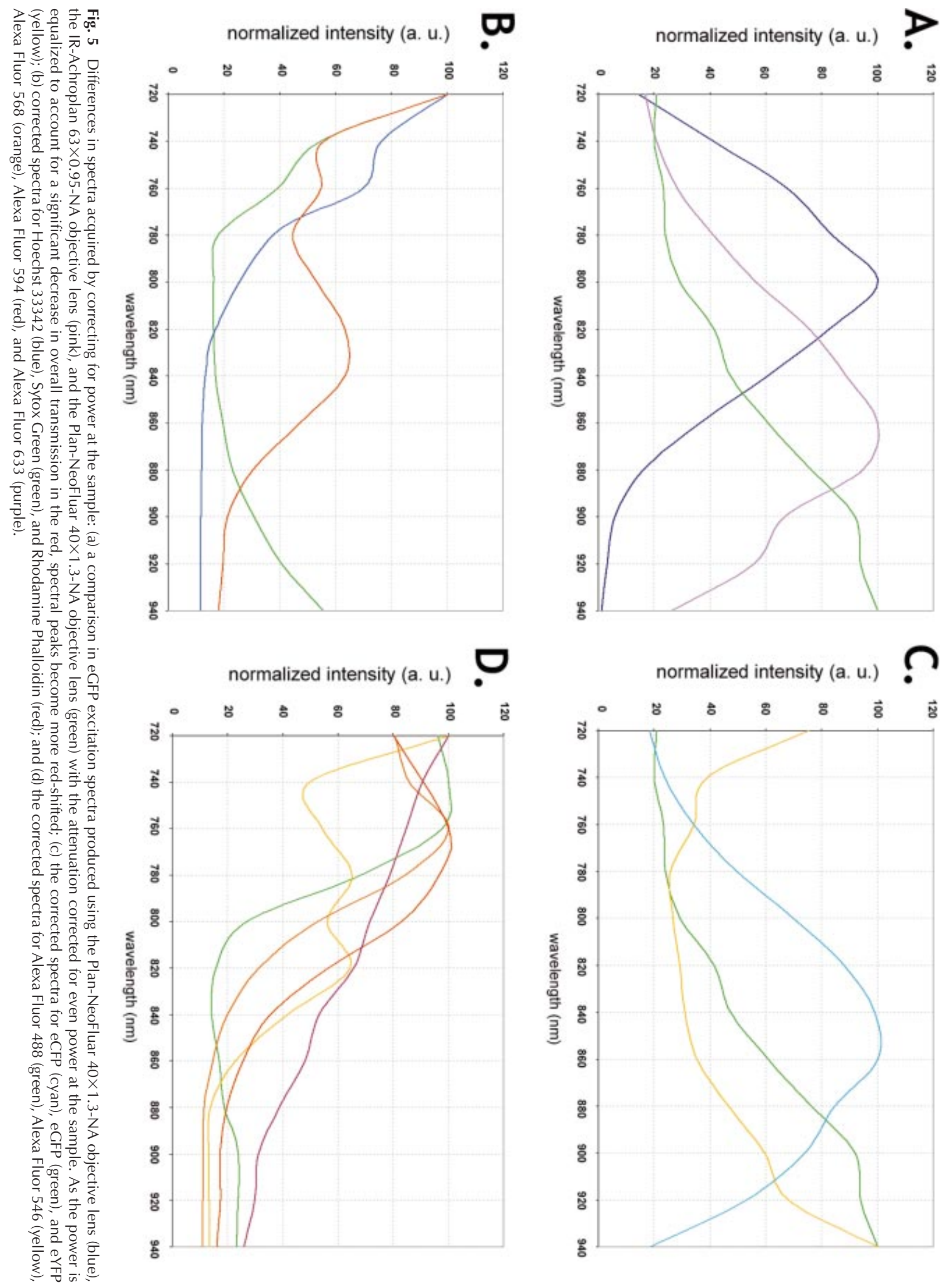
With power normalized at the sample, excitation spectra are quite different from nonnormalized spectra. Figure 5(a) (see Color Plate 3) compares the excitation spectra measured for eGFP with and without correction. The blue curve shows eGFP fluorescence as the laser is tuned from 720 to $940 \mathrm{~nm}$ using the Zeiss Plan-NeoFluar $40 \times / 1.3-N A$ objective lens. The pink curve is the eGFP spectra taken from 720 to $940 \mathrm{~nm}$ using the IR-Achroplan $63 \times / 0.95-N A$ water immersion objective lens. This lens has more even transmission over this range (see Ref. 15), and as a result, the eGFP excitation peak shifts more into the red, but is still biased by lower power output from the laser over this range. The green curve in Fig. 5(a) shows the eGFP spectra taken from 720 to $940 \mathrm{~nm}$ using the Plan-NeoFluar $40 \times 1.3-N A$ objective lens but correcting for power at the sample. With this correction, the peak excitation wavelength is clearly red-shifted as compared to the biased value. The corrected spectrum more closely matches previous data acquired using more quantitative methods to measure the cross-section value ${ }^{10,12}$ of eGFP.

With this data in hand, we then proceeded to acquire corrected excitation spectra for several commonly used fluorochromes. The eCFP, eGFP and eYFP spectra are shown in Fig. 5(c); Sytox Green, Hoechst 33342 and Rhodamine Phalloidin spectra are shown in Fig. 5(b); and several dyes from the Alexa Fluor series are shown in Fig. 5(d). In some cases, we suspect that some of these dyes may have significant singlephoton excitation in this range that may contribute to our data because of the strong response of some fluorochromes at low wavelengths. Interestingly, many of the Alexa Fluor compounds have Rhodamine-like structures, yet have multiphoton excitation peaks that are more blue-shifted than Rhodamine.

\section{Conclusion}

The results show that multiphoton excitation spectra are useful for excitation fingerprinting and that it is possible to obtain realistic multiphoton excitation spectra using a laser scanning microscope. Here, we have used the software-tunable Coherent Chameleon laser coupled to the LSM 510 META NLO to acquire $x-y$ images of biological samples at multiple excitation wavelengths, creating excitation lambda stacks. The mean intensity of pixels within the image plotted versus excitation wavelength reveals the excitation spectra. The excitation lambda stacks can be separated into individual images based on linear unmixing using these excitation spectra. Thus, we have added a new tool for fluorescence crosstalk elimination for multiphoton excitation. This approach does not depend on the use of a particular detector and excitation lambda stacks can be acquired using nondescanned detectors, enabling more sensitive detection of scattered photons in deep tissue combined with the most advanced methods for crosstalk separation. Although $x-y$ images are collected sequentially as the laser tunes to different wavelengths, 12 images were acquired at wavelengths all across the tunable spectrum of the laser in less than 2 min, and in many cases it may not be necessary to collect data from as many wavelengths as shown here.

Excitation fingerprinting complements and extends the capabilities currently offered using the META detector for emission fingerprinting, adding to the arsenal of tools available for spectral flexibility. We show here that excitation and emission fingerprinting can yield similar results using dyes such as eGFP and Alexa Fluor 488 and that dyes that cannot be reliably separated using emission fingerprinting, such as Sytox Green, Fluorescein, and eGFP, can be faithfully unmixed based on their multiphoton excitation spectra. Interestingly, this may also have implications for fluorescent resonance energy transfer (FRET), particularly when using CFP and YFP. It is clear from our corrected data that CFP and YFP have very distinct excitation spectra, ideal for excitation fingerprinting. The clear separation of the excitation spectra has already been useful for eliminating spectral crosstalk as some investigators choose to excite CFP at or near $800 \mathrm{~nm}$, where there is minimal multiphoton excitation crosstalk. ${ }^{16-18}$ We are currently investigating the use of excitation fingerprinting in FRET studies.

In addition to showing that excitation fingerprinting can be used to eliminate spectral crosstalk, we also demonstrated that nonbiased excitation spectra can be acquired to provide realistic information about multiphoton excitation maxima. This greatly aids in choosing the best excitation wavelength for imaging samples with multiple fluorescent labels. The comparison of the biased and nonbiased data enable the best compromise to be made between the available power at the sample and the excitation maxima of the dye. This is particularly important in experiments where multiple dyes are used. Also, by directly measuring the excitation of autofluorescent species, one can also quickly determine if it is possible to avoid or minimize this signal by choosing another excitation wavelength or if linear unmixing will be necessary. In addition to experimental optimization, generating multiphoton excitation spectra directly from biological samples enables one to study environmental factors that influence nonlinear excitation events. Multiphoton microscopy is still in its infancy and many groups are engaged in dye optimization by testing structural permutations. ${ }^{19,20}$ The tools presented here provide a rapid method for determining the compatibility of new compounds with biological media and for determining the best excitation criteria for new dyes.

\section{Acknowledgments}

We are grateful to Coherent Inc., Santa Clara, CA and APE, Berlin, Germany for the use of the Chameleon laser and the Carpe autocorrelator, respectively. We thank Rusty Lansford for the gift of H2B-GFP expressing virus and Diane Olson for help in preparing the manuscript. The authors thank NSF (BES-0086944) and NIH (HD 37105) for support.

\section{References}

1. W. Denk and K. Svoboda, "Photon upmanship. Why multiphoton imaging is more than a gimmick," Neuron 18, 351-357 (1997).

2. D. W. Piston, "Imaging living cells and tissues by two-photon excitation microscopy," Trends Cell Biol. 9, 66-69 (1999).

3. R. Lansford, G. Bearman, and S. E. Fraser, "Resolution of multiple green fluorescent protein color variants and dyes using two-photon microscopy," J. Biomed. Opt. 6, 311-318 (2001).

4. M. E. Dickinson, G. Bearman, S. Tille, R. Lansford, and S. E. Fraser, "Multi-spectral imaging and linear unmixing adds a whole new dimension to laser scanning fluorescence microscopy," BioTechniques 31, 1272-1279 (2001).

5. M. E. Dickinson, C. W. Waters, R. Wolleschensky, G. Bearman, S. Tille, and S. E. Fraser, "Sensitive imaging of spectrally overlapping fluorochromes using the LSM 510 META," in Multiphoton Micros- 
copy in the Biomedical Sciences, A. Periasamy and P. T. So, Ed., Proc. SPIE 4620, 123-136 (2002).

6. T. Haraguchi, T. Shimi, T. Koujin, N. Hashiguchi, and Y. Hiraoka, "Spectral imaging fluorescence microscopy," Genes Cells 7, 881887 (2002).

7. R. Nashmi, M. E. Dickinson, S. McKinney, M. Jareb, C. Labarca, S. E. Fraser, and H. A. Lester (submitted for publication).

8. C. Xu and W. W. Webb, "Measurement of two-photon excitation cross-sections of molecular fluorophores with data from $690 \mathrm{~nm}$ to 1050 nm," J. Opt. Soc. Am. B 13, 481-491 (1996).

9. J. B. Shear, C. Xu et al., "Multiphoton-excited visible emission by serotonin solutions," Photochem. Photobiol. 65, 931-936 (1997).

10. C. Xu, "Two-photon cross sections of indicators," in Imaging Neurons: A Laboratory Manual, R. Yuste, F. Lanni, and A. Konnerth, Eds., Cold Spring Harbor Laboratory Press, Cold Spring Habor, NY (2000).

11. F. Bestvater, E. Spiess, G. Stobrawa et al., "Two-photon fluorescence absorption and emission spectrum of dyes relevant for cell imaging," J. Microsc. 208, 108-115 (2002).

12. W. Zipfel, unpublished data, see http://cellscience.bio-rad.com/ products/multiphoton/Radiance $2100 \mathrm{MP} / \mathrm{mpspectra} \cdot \mathrm{htm}$

13. K. J. Longmuir, S. M. Haynes, M. E. Dickinson, A. J. Waring, and J. C. Murphy, "Optimization of a peptide/non-cationic lipid gene delivery system for effective microinjection into chicken embryos in vivo," Mol. Therapy 4, 67-74 (2001).

14. W. Denk, J. H. Strickler, and W. W. Webb, "Two-photon laser scanning microscopy," Science 248, 73-76 (1990).
15. R. Wolleschensky, M. E. Dickinson, and S. E. Fraser, "Group velocity dispersion and fiber delivery in multiphoton laser scanning microscopy," in Confocal and Two-Photon Microscopy: Foundations, Applications and Advances, A. Diaspro, Ed. Wiley-Liss, New York (2001).

16. G. Y. Fan, H. Fujisaki, A. Miyawaki, R.-K. Tsay, R. Y. Tsien, and M. H. Ellisman, "Video-rate scanning two-photon excitation fluorescence microscopy and ratio imaging with yellow chameleons," Biophys. J. 76, 2412-2420 (1999).

17. V. J. LaMorte, T. B. Krasieva, A. Zoumi, B. M. Forman, and C. Solier, "One-photon versus two-photon excited fluorescence resonance energy transfer of GFP fusion proteins," in Multiphoton Microscopy in the Biomedical Sciences, A. Periasamy and P. T. So, Eds., Proc. SPIE 4620, 73-78 (2002).

18. M. Elangovan, H. Wallrabe, Y. Chen, R. N. Day, M. Barroso, and A. Periasamy, "Characterization of one-and two-photon excitation fuorescence resonance energy transfer microscopy," Methods 29, 58-73 (2003).

19. M. Albota, D. Beljonne et al., "Design of organic molecules with large two-photon absorption cross sections," Science 281, 16531656 (1999).

20. M. Rumi, J. E. Ehrlich et al., "Structure-property relationships for two-photon absorbing chromophores: bis-donor diphenylpolyene and bis(styryl)benzene, derivatives," J. Am. Chem. Soc. 122, 9500-9510 (2000). 